\title{
Student Perspectives on Indigenous Health Content in Pre-Clinical Medical Education
}

\author{
Marissa Ley ${ }^{1}$, MA; Heather Castleden², PhD; and Debbie Martin³, PhD \\ 1School of Medicine, Dalhousie University \\ 2Department of Geography, Queen's University \\ ${ }^{3}$ Department of Health and Human Performance, Dalhousie University
}

DOI: https://doi.org/10.15273/hpj.v1i2.10650

\begin{abstract}
Introduction: In 2015, the Truth and Reconciliation Commission of Canada released 94 calls to action. These calls were intended to redress harms inflicted on Indigenous peoples as a result of the Indian Residential School system and to advance the process of reconciliation of Canada. Several of these calls to action are directed specifically toward educating health-care providers as a means to actively eliminate racism in health-care experiences for Indigenous peoples. Objectives: To identify the learning needs of pre-clerkship medical students with respect to Indigenous health content and curriculum, and to explore the perspectives of pre-clerkship medical students on existing gaps in the medical curriculum regarding Indigenous health. Methods: This study involved semi-structured interviews with 14 first- and second-year (pre-clinical/pre-clerkship) medical students at one medical school. Thematic analysis was performed using NVivo data management software to identify common themes, and then considered within the context of the existing literature. Results: Three main themes were identified: (a) Familiarity with Indigenous culture prior to medical school, (b) constructive criticism about Indigenous education in medical school, and (c) pervasive lack of education about Indigenous health issues in the program. Discussion: Participants felt they needed more time devoted to learning about Indigenous peoples' health, as there are many topics to explore. Most participants felt that their understanding of health issues impacting Indigenous peoples has not been sufficient in their primary, secondary, and post-secondary education to date and offered suggestions for improving the Indigenous health content in the medical school curriculum. These suggestions included providing the education earlier in the program, having Indigenous peoples involved in content creation and delivery, and ensuring all learners have sufficient opportunity to learn more about Indigenous peoples and their health. Conclusion: Through this research we gain a glimpse of how future medical providers are interpreting Indigenous health curriculum, and whether and how they intend to apply this education to their own future practice.
\end{abstract}

NOTE: While the term Aboriginal has been used in Canada and Australia, the term Indigenous is used in this paper as an all-encompassing term to speak of the diverse cultures and peoples who are the original inhabitants of Canada, as it is used globally (Indigenous Foundations, 2009). 


\section{Student Perspectives on Indigenous Health Content in Pre-Clinical Medical Education}

The authors acknowledge that this research and writing has taken place on the unceded and ancestral territory of the Mi'kmaq. We pay our respects to these lands and waters and honour the treaties of Peace and Friendship.

In Canada, Indigenous peoples' experiences with health care are shaped by what is referred to as the double burden of racism and colonialism (Yeung et al., 2018). The 2015 Truth and Reconciliation Commission of Canada (TRC) has provided 94 calls to action to support reconciliation between Indigenous and nonIndigenous peoples in Canada, addressing the intergenerational trauma of the Indian Residential School (IRS) system. The TRC determined that removing barriers faced by Indigenous peoples when accessing health care at all levels (provider, institutional, and system) would positively influence the health opportunities available for Indigenous peoples (McNally \& Martin, 2017). In response to this, a number of medical schools in Canada have implemented mandatory curriculum or community-based activities that focus on Indigenous peoples' health issues as a way to improve the knowledge, skills, and attitudes necessary to shift away from racist and colonialist assumptions (Yeung et al., 2018; Zhou et al., 2012). However, we know very little about whether this type of curriculum is meeting the needs of medical students, and what their perceptions are regarding the curriculum they are receiving.

This study used thematic analysis to analyze semi-structured, qualitative interview data from medical school students in order to begin assessing both the education delivered to future health-care providers about Indigenous peoples and their health and the extent to which the TRC recommendations were being followed. In-depth interviews with pre-clerkship (firstand second-year) medical students at one school were used to (a) identify their learning needs with respect to Indigenous health content in the medical curriculum, and (b) determine their perspectives on existing gaps in the medical curriculum in this regard. The goals of this study are to identify, document, and understand firstand second-year medical students' current knowledge, skills, and attitudes toward Indigenous health.

\section{Background}

In 2016, Indigenous peoples (First Nations, Inuit, Métis) constituted $4.9 \%$ of the total Canadian population, reflecting an increase from 3.8\% in 2006 (Statistics Canada, 2017). The Indigenous population in Canada has been shown to be growing at a rate that is four times faster than non-Indigenous Canadians, which is thought to be a result of higher birth rates and increased self-identification compared to previous generations (Statistics Canada, 2017). Indigenous peoples in Canada continue to experience persistent health disparities, including a lower life expectancy and increased risk of heart disease, diabetes, obesity, sexually transmitted infections, and suicide (Jacklin et al., 2014). There are various contributing factors for these health disparities: loss of culture, issues of self-governance, colonialism (historically and today), child apprehensions and out-of-culture adoptions by the state, and the residential school experiences many individuals and communities were exposed to and continue to experience today through intergenerational trauma (Wilson et al., 2018). Intergenerational trauma is a cumulative effect of IRS trauma that undermines the health and well-being of Indigenous peoples today (Bombay et al., 2014).

Given these issues, it is crucial that medical students obtain appropriate education and training that is well informed and patient centred so that they can develop the necessary skills to navigate health-care issues confronting Indigenous patients. It is important for future health-care providers to learn about the underlying communication issues that can adversely affect the patient-physician encounter and how best to circumvent these, building confidence in their skill to deliver culturally-sensitive medical care, which is a basic human right (Yeung et al., 2018). Cultural 
sensitivity in health-care encompasses understanding one's own culture, having open and compassionate communication, and listening to patients and families to better understand and incorporate their beliefs and values into their health-care management (Brooks et al., 2019). Cultural safety in healthcare refers to a safe environment for people of all cultures and backgrounds, where their values and needs are understood and respected (Williams, 1999).

Encouraged and supported by the Canadian Medical Association (CMA), most universities in Canada have been working to expand their respective curricula to incorporate Indigenous health courses (Jacklin et al., 2014). The CMA Board of Directors has highlighted the importance of recognizing and addressing the impact of intergenerational trauma caused by the IRS system and supports the implementation of the TRC calls to action for medical learners and practising professionals (CMA Board of Directors, 2015, as cited in HealthCareCAN, 2016). Call to action \#23 addresses the need for cultural competency training for all health-care professionals, and call to action \#24 calls upon medical schools to require students to take a course about Indigenous health issues, the history and legacy of the IRS system, and other determinants of health impacting Indigenous peoples today (Truth and Reconciliation Commission of Canada, 2015).

It has been documented that medical students seem to be aware of "Indigenous health issues" generally, but they often have a more limited understanding of the socio-cultural and economic influences on these issues, as well as the intersectional way in which diverse factors such as poverty and racism may combine to affect the health of patients (Zhou et al., 2012). Without a nuanced understanding of the interrelationships between structural determinants that affect the health of Indigenous peoples, it is unlikely that knowledge, skills, and behaviours will be fundamentally altered (Yeung et al., 2018), which raises questions as to whether simply increasing Indigenous content in medical curriculum is sufficient. To date, there has been limited research to assess whether medical students feel that the education they are receiving is effective in transforming their ability and willingness to work with Indigenous patients in a culturally safe way. One issue is that medical students' knowledge about Indigenous peoples remains rooted in personal biases and negative medical and educational portrayals, which stems from the historical and ongoing effects of colonialism and anti-Indigenous racism in Canada and around the world (Ly \& Crowshoe, 2015). This is also known as the "hidden curriculum," and many medical schools are attempting to reduce the biases that the hidden curriculum creates among their learners (Kidman et al., 2013). The first step in challenging these biases is for the learner to become aware of their own biases, learn about bias in the medical system, and be taught ways in which they can work toward reducing bias in their lives and practice.

\section{Study Objectives}

The purpose of the study was to address the following specific research objectives: (a) Identify the learning needs of pre-clerkship medical students with respect to Indigenous health content and curriculum, and (b) explore the perspectives of pre-clerkship medical students on existing gaps in the medical curriculum regarding Indigenous health.

\section{Methodology}

In this research study, the student researcher was non-Indigenous, but had previously completed an MA where the thesis research was done with an Indigenous community using narrative inquiry and TwoEyed Seeing. The student researcher has also been part of several research projects and groups relating to Indigenous health promotion. The research supervisor is an Indigenous researcher. These experiences were used to establish the research questions, and to form questions and probing questions for the interview guide.

This exploratory study was guided by Interpretative Phenomenological Analysis, and 
thematic analysis. Interpretative Phenomenological Analysis is a methodology that explores how participants derive meaning from their lived experiences (Pietkiewicz \& Smith, 2014). It incorporates elements of phenomenology and other qualitative research methods, and involves self-reflection on the part of the participants and iterative reflection and immersion in the data on the part of the researcher. Phenomenology is a qualitative approach that is used to explore and find meaning from the lived experiences of a specific group of people (Hall et al., 2016).

Data was collected using semistructured interviews and analyzed using thematic analysis (Braun \& Clarke, 2021). The call for participants was done via a one-slide presentation with the study details and contact information for the lead researcher. Participants were selected according to the following shared traits: (a) enrolled in one specific university's MD program, and (b) are first- or second-year medical students. This population was selected because their learning during pre-clerkship years is done mainly in the classroom and is standardized for all students in the class, which is not the case for clerkship training in years 3 and 4. Participants included 14 medical students (six in first year; eight in second year). Institutional Review Board approval was obtained, and informed consent was obtained before data collection commenced.

Semi-structured interviews were used to gain an in-depth understanding of medical students' perspectives. This interview guide was piloted with three students in their third year of medicine, who were ineligible for the study but had been taught using similar lectures and sessions. The following is an example question from the interview guide, along with probing questions for follow up:

1) What training/education have you received (if any) regarding Indigenous health? [Probes: Ask about formal education in classrooms/research, but also about informal learning opportunities: lived experiences, volunteering, interest groups, public lectures/events, etc.]
The interviews were conducted in person and audio-recorded at the university in a privately booked study room. An appropriate number of participants were interviewed, and study objectives were deemed to have been met. Recruitment was then ceased, and no further interviews were done. After the interview responses were transcribed, coding and thematic analysis were performed using NVivo data management software. Here, the goal was to identify themes within individual participant responses and within the entire data set using thematic analysis. Thematic analysis is an approach to identifying, analyzing, and reporting qualitative data and the patterns, or themes (Braun \& Clarke, 2006). There are six phases of conducting thematic analysis, which are as follows:

1. Familiarizing yourself with the data,

2. Generating initial codes,

3. Searching for themes,

4. Reviewing themes,

5. Defining and naming themes, and

6. Producing the report.

The resultant themes were then considered from multiple angles and discussed in the context of the existing literature.

\section{Findings}

Three major themes were derived upon analysis of the interviews. They are related to the participants' previous and current knowledge of Indigenous peoples and their health, the students' perception of their medical school's Indigenous health curriculum, and the pervasive lack of education about Indigenous health issues.

\section{Familiarity with Indigenous Culture Prior to Medical School}

Most participants said that, at one point in their lives, they had lived in close proximity to an Indigenous community. None self-identified as being Indigenous. For instance, one participant notes that they went to high school with First Nations people from a nearby community, and that some of the high school 
curriculum was shaped by an Indigenous teacher:

There was a reserve close to my high school, and their students went to my high school. That would be my only real experience with an Aboriginal community. They would be in our classes and there was actually a Mi'kmaw teacher-one of the teachers was Mi'kmaw. He actually started a Mi'kmaq studies class when I was in my grade 12 year. He was really cool. - First-Year Student

Although many participants in the study noted that they may have encountered Indigenous peoples during high school, this did not necessarily mean that the interactions led to strong cross-cultural relationships or that they were free from racism.

My high school was a little racist. For example, there was one girl, a White girl, who was dating a First Nations boy, and everyone kind of made fun of her for that and called her bad names. It was also very much like, if you look in the cafeteria you can see Native kids at one table, you know, sitting separated like that from the other kids. - First-Year Student

Without being provided historical background and context, the lived experiences of the participants in the study may not have offered opportunities to break down stereotypes or to understand the barriers faced by Indigenous peoples. As the next participant describes, the first time they were exposed to Indigenous issues was during medical school.

I think a lot of people haven't been exposed to Aboriginal health care issues before medicine at all. I was an interviewer this year for [Medical School] and I had a question surrounding Aboriginal health and I was blown away at how little some people knew. I would probably have been in the same situation. I never intentionally learned about it in high school or before med school. Second-Year Student
As the participant explains, while questions about Indigenous health may be included in the interview for incoming medical students, the interviewer or actor may not have a deep understanding of the topic, despite being in medical school themselves.

\section{Indigenous Education in Medical School- Constructive Criticism}

The second major theme pertains to the content and delivery of Indigenous health lessons in the medical school curriculum. The participants had differing perspectives about this. For example, many participants discussed the sessions dedicated to Indigenous health in the curriculum in a positive way:

Well, we had two weeks of pro-comp [Professional Competencies is a course that is delivered once each week] dedicated to Aboriginal health. Both sessions were mostly led by Aboriginal people and then we had each lecture followed by two hours of, like, a normal pro-comp tutorial. But we also had Aboriginal coach tutors. Every group had an Aboriginal community member in their group sessions. I think they [the sessions] went really well, at least a lot of them ... I think a lot of people did learn a lot from them. - Second-Year Student

We can see from the next participant, however, that the effectiveness of these sessions is subjective to each student or group of students. While they agreed on number of days in which Indigenous health content was delivered, one of the participants noted that they felt the Indigenous content was not embedded into other curriculum content areas, making it feel siloed:

[We] only get a day or two days for Indigenous health, and then we have a day of Refugee health, ... and it's like we have these days that are pretty siloed, even though they're all part of the [same] course, and most is in second year. Second-Year Student 
Another participant makes a strong case for starting the Indigenous content earlier in the medical school curriculum, and this was brought up by many participants. It is worth noting, however, that this institution's curriculum has made changes since these data were collected in 2018, with this curriculum now being offered earlier. As described in the TRC calls to action, medical schools have a duty to introduce this new aspect of the curriculum in the right way so that students are able to build upon skills of cultural competency throughout their education.

We can't assume that everyone knows [about] Aboriginal health. And certainly, things like the residential schools and how reserves are structured, how health care is provided and funded to Indigenous populations, to drug coverage and access, which are all very different for Indigenous peoples. Those are all things that really need to be laid out and given to us in a direct, explicit sort of way, early on. First-Year Student

\section{The Lack of Education About Indigenous Health Issues is Pervasive}

Medical students, much like the general population, have not had adequate education about Indigenous history and health issues in primary school, secondary school, or during their undergraduate education. Many students are encountering these concepts and learning about the social determinants of health that impact Indigenous health for the first time in medical school, which is not soon enough for some patient encounters.

It's strange we get to see patients for a year, or actually a year and a half, as medical students without having, you know, a basic understanding of who lives in our province/region and how to be culturally competent. - Second-Year Student

As described in the TRC calls to action, medical schools are to teach their students about Indigenous health issues, the history and legacy of the IRS system, and other determinants of health impacting Indigenous peoples today. The goal of introducing students to these concepts is to create future health-care providers who are able to deliver culturally safe care to all of their patients.

There is also an element of reconciliation that universities have a duty to provide that by making space in the curriculum for these issues. That is one way that universities, which are a relic of colonialism and privilege in the Americas, can make steps towards reconciling. These are things that graduates need to learn because they will make them better doctors and better able to provide for their patients. That's one thing, and then two, it is a moral ethical obligation to educate students and doctors on these issues as a means of reconciliation. Second-Year Student

The TRC call to action \#23 also calls for an increased number of Indigenous health-care providers to be trained, and this need was noticed by participants in the lack of diversity observed in their instructors.

I really don't feel like it's been presented well at all. You know, even if you look at our lecturers it has been a lot of very White people, and even the diversity among lecturers or tutors that we have seen is not nearly enough. I haven't met a single First Nations person either as a lecturer or preceptoryet, a year in. It's not only the content that's lacking but also the representation. - Second-Year Student

As participants began to learn about the historical and current injustices and health disparities faced by Indigenous peoples, they noted feeling frustrated about how little they know about such an important issue. The result of this frustration is that some were finding creative solutions to supplement their existing curriculum with additional learning opportunities, such as student-led organizations and volunteer opportunities. 
I would say I've had very little education around Indigenous health in any sort of formal way. In the first-year med school curriculum it's touched on a little bit, but it feels rather tokenistic ... There have been opportunities to learn about it outside of the classroom. So, I participate in the Indigenous Health Interest Group and there have been opportunities to engage with health issues there that have been very valuable. - First-Year Student

\section{Discussion}

The findings in this study relate to medical students' knowledge about, and current and past education relating to, Indigenous health in Canadian medical schools. While participants were keen to learn more about Indigenous health, there has been limited opportunities for them to do so in a meaningful way. Similar to Sylvestre et al. (2019), the participants in this study agree that without ample opportunity to interact with Indigenous patients and to learn about how structural determinants interact to affect the health of Indigenous peoples, there is concern among students that they risk reinforcing antiIndigenous racism.

While many participants had some experiences with and education regarding Indigenous health issues before entering medical school, these were not always positive. Students expressed awareness of the individual racism Indigenous people encounter but recognized that they had little intentional learning on Indigenous history, culture, and health issues or the structural and systemic racism embedded in colonial education and health care systems. This theme of familiarity with Indigenous cultures is important for predicting students' future interest in developing their practices (Margolius \& Bodenheimer, 2010). It is imperative that medical practitioners be equipped with more than adequate knowledge and competence to address the health-care needs of Indigenous peoples in Canada. Having Indigenous scholars and community members create and deliver the curriculum is one way in which this gap in previous education can be addressed.

Participants felt that two days of Indigenous content in second year is not enough. Students generally found the Indigenous curriculum delivery to be a positive experience, but also limited in how it is integrated into other aspects of curriculum and how it is framed and built upon over the course of the two-year preclerkship curriculum. As Jacklin et al. (2014) notes, there are significant challenges in providing "new" education to medical students-largely in the negotiation of curricular time. Additionally, there is a significant need to recruit Indigenous healthcare professionals and educators to develop and deliver this content to students (Zhou et al., 2012). Participants noted that the lack of education about Indigenous health is not limited just to medical students but is pervasive within Canadian society. As such, it is imperative that the education one receives in medical school addresses this learning gap, so that future providers are well informed and able to be competent physicians for all of their future patients. It is recommended that medical schools ensure they are introducing these topics earlier and more regularly to students, as they then gain time and opportunities with which to practice their skills, as well as explore elective and other learning opportunities relating to Indigenous health and well-being throughout their training, aided by early exposure to these topics and communities. Medical students are also then better prepared to combat pre-existing stereotypes that students may have and create more culturally-aware and safe practitioners (Ly \& Crowshoe, 2015). Some students with a pre-existing interest in Indigenous health are seeking out their own extra-curricular learning opportunities to supplement the curriculum. However, this means that students without this interest, who may know very little about Indigenous peoples and their health, are not exposed to these opportunities and therefore have very few opportunities to counter preexisting stereotypes and assumptions. 


\section{Study Limitations}

Limitations to this study include the possibility that the students who volunteered to participate may have an interest in Indigenous health and therefore may not be representative of the entire medical school class. Another limitation is that the lead researcher was known to the participants, and this may have altered the responses provided to be more socially acceptable.

Since these data were collected, there have been significant changes made to the Indigenous health content in the medical school curriculum, which were created and delivered by an Indigenous professor. A comparative study evaluating students' perspectives during their education and at graduation could be used to compare the relative efficacy of this new content.

\section{Conclusion}

In this study, we found that among preclerkship medical students, Indigenous health is a topic seen as important for the future practice of physicians and one that needs to be improved upon within the medical school curriculum. Since the TRC calls to action include the education of future health-care providers, this study sought to document and explore existing students' perspectives on Indigenous health in one medical school's curriculum and offer suggestions for improving the experience for students. We also found that many students had some familiarity with Indigenous cultures prior to medical school, but that this was often inadequate and not based on formal education on the subject. Students were able to evaluate the education they had received thus far in medical school regarding Indigenous health, and they had suggestions for improvement. The lack of education about Indigenous health issues is a pervasive issue that is best addressed with several interventions: providing quality education earlier in the medical school curriculum as part of reconciliation, having Indigenous peoples involved in developing and teaching this content, and ensuring all medical students are exposed to informative and engaging Indigenous health learning opportunities.

\section{References}

Bombay, A., Matheson, K., \& Anisman, H. (2014). The intergenerational effects of Indian residential schools: Implications for the concept of historical trauma. Transcultural Psychiatry, 51(3), 320-338. https://doi.org/10.1177\%2F13634615 13503380

Braun, V., \& Clarke, V. (2006). Using thematic analysis in psychology. Qualitative Research in Psychology, 3(2), 77-101.

Braun, V., \& Clarke, V. (2021). One size fits all? What counts as quality practice in (reflexive) thematic analysis? Qualitative Research in Psychology, 18(3), 328-352. https://doi.org/10.1080/14780887.20 20.1769238

Brooks, L. A., Manias, E., \& Bloomer, M. J. (2019). Culturally sensitive communication in healthcare: A concept analysis. Collegian, 26(3), 383-391. https://doi.org/10.1016/j.colegn.2018. 09.007

Hall, E., Chai, W., \& Albrecht, J. A. (2016). A qualitative phenomenological exploration of teachers' experience with nutrition education. American Journal of Health Education, 47(3), 136-148. https://doi.org/10.1080/19325037.20 16.1157532

HealthCareCAN (2016). Issue brief: Truth and Reconciliation Commission of Canada: Health-related recommendations. https://www.healthcarecan.ca/wpcontent/themes/camyno/assets/docu ment/IssueBriefs/2016/EN/TRCC_EN.p df

Indigenous Foundations (2009). Terminology: So, which terms do I use? First Nations \& Indigenous Studies, University of British Columbia. https://indigenousfoundations.arts.ubc. $\mathrm{ca} /$ terminology/ 
Jacklin, K., Strasser, R., \& Peltier, I. (2014). From the community to the classroom: The Aboriginal health curriculum at the Northern Ontario School of Medicine. Canadian Journal of Rural Medicine, 19(4), 143-150.

Kidman, J., Yen, C.-F., \& Abrams, E. (2013). Indigenous students' experiences of the hidden curriculum in science education: A cross-national study in New Zealand and Taiwan. International Journal of Science and Mathematics Education, 11(1), 43-64. https://doi.org/10.1007/ s10763-012-9365-9

Ly, A., \& Crowshoe, L. (2015). 'Stereotypes are reality': Addressing stereotyping in Canadian Aboriginal medical education. Medical Education, 49(6), 612-622. https://doi.org/10.1111/medu.12725

Margolius, D., \& Bodenheimer, T. (2010). Transforming primary care: From past practice to the practice of the future. Health Affairs, 29(5), 779-784. https://doi.org/10.1377/hlthaff.2010.0 045

McNally, M., \& Martin, D. (2017). First Nations, Inuit and Métis health: Considerations for Canadian health leaders in the wake of the Truth and Reconciliation Commission of Canada report. Healthcare Management Forum, 30(2), 117-122. https://doi.org/10.1177/ 0840470416680445

Pietkiewicz, I., \& Smith, J. A. (2014). A practical guide to using interpretative phenomenological analysis in qualitative research psychology. Psychological Journal, 20(1), 7-14.

Statistics Canada. (2017, October 25). Aboriginal peoples in Canada: Key results from the 2016 Census. https://www150. statcan.gc.ca/n1/daily-quotidien/ 171025/dq171025a-eng.htm

Sylvestre, P., Castleden, H., Denis, J., Martin, D., \& Bombay, A. (2019). The tools at their fingertips: How settler colonial geographies shape medical educators' strategies for grappling with AntiIndigenous racism. Social Science \& Medicine, 237, Article 112363. https://doi.org/10.1016/j.socscimed.20 19.112363

Truth and Reconciliation Commission of Canada (2015). Truth and Reconciliation Commission of Canada: Calls to action. https://ehprnh2mwo3.exactdn.com/w p-Content/uploads/2021/01/ Calls_to_Action_English2.pdf

Williams, R. (1999). Cultural safety-what does it mean for our work practice? Australian and New Zealand Journal of Public Health, 23(2), 213-214. https://doi.org/10.1111/j.1467842X.1999.tb01240.x

Wilson, N., Ewen, S., \& Mazel, O. (2018). Medical students in Aboriginal Community Controlled Health Services: Identifying the factors involved in successful placements for staff and students. Australian Medical Student Journal, 8(2), 26-30. http://www.amsj.org/archives/6330

Yeung, S., Bombay, A., Walker, C., Denis, J., Martin, D., Sylvestre, P., \& Castleden, H. (2018). Predictors of medical student interest in Indigenous health learning and clinical practice: A Canadian case study. BMC Medical Education, 18(1), Article 307. https://doi.org/10.1186/ s12909-018-1401-1

Zhou, A. W., Boshart, S., Seelisch, J., Eshaghian, R., McLeod, R., Nisker, J., Richmond, C. A. M., \& Howard, J. M. (2012). Efficacy of a 3-hour Aboriginal health teaching in the medical curriculum: Are we changing student knowledge and attitudes? Health Education Journal, 71(2), 180188. https://doi.org/10.1177\% 2F0017896910394544 